\title{
Desarrollo y movilidad social. Análisis comparado entre Andalucía y Cataluña
}

\author{
Félix Requena Santos \\ Universidad de Málaga. Área de Sociología \\ frequena@uma.es
}

Recibido: 23-01-2008

Aceptado: 09-06-2008

\section{Resumen}

En este trabajo se estudian los procesos de movilidad social en función del desarrollo que tienen las sociedades. Para ello se han escogido dos momentos en el tiempo (1999 y 2004) y dos lugares en el espacio (Andalucía y Cataluña). De esta forma, se pueden realizar análisis comparados tanto de forma longitudinal, comparando dos momentos en los procesos de desarrollo, como comparaciones transversales entre las dos comunidades elegidas. La tesis principal que se va ha seguir en este artículo es comprobar que en las sociedades avanzadas los procesos de movilidad son más fuertes e intensos que en las sociedades menos desarrolladas. Se trata de ver si dicha proposición se confirma en las sociedades objeto de nuestro análisis en los sucesivos momentos estudiados. Los resultados han puesto de manifiesto que los procesos de movilidad social no funcionan de la misma forma en unas sociedades u otras, y que no siempre se cumple la hipótesis de referencia.

Palabras clave: movilidad social, desarrollo, Andalucía, Cataluña, estructura comparada.

Abstract. Development and social mobility. Comparative analysis between Andalusia and Catalonia

This article focuses on the study of trends in the processes of social mobility in terms of development of societies. Two moments in time (1999 and 2004) and two locations in space (Andalusia and Catalonia) were analysed. Longitudinal comparative analysis comparing two different times in development processes, and cross-comparisons between the two communities, Andalusia and Catalonia, were realised. The main argument is that in advanced societies mobility processes are stronger and more intense than in less developed societies. The results show that the processes of social mobility don't work in the same way in all the societies, and that the reference hypothesis is not always confirmed.

Key words: Social Mobility, Development, Andalusia, Catalonia, Comparative Structure.

\section{Sumario}

1. Introducción

2. Movilidad intergeneracional y desarrollo: hipótesis

3. Datos, variables y método
4. Hallazgos empíricos

5. Conclusiones

Referencias bibliográficas 


\section{Introducción}

La movilidad social es el cambio de posiciones sociales que, a través del tiempo, se produce en una estructura social. Estos cambios se estudian comparando las distribuciones estructurales en diferentes momentos. La movilidad social es, pues, un proceso por el cual las personas se desplazan entre la estructura social, y en el que la estructura misma se transforma. Para el análisis de la movilidad social, se usan fundamentalmente los grupos o las categorías ocupacionales (Erikson y Goldthorpe, 1992; Carabaña, 1997 y 1999; Gobernado, 1996; Requena, 2005).

En las estructuras sociales, existen diversos procesos que tienen un efecto directo sobre la movilidad social. Estos procesos son similares en los distintos países avanzados. Nos referimos, por ejemplo, a los siguientes: el número de vacantes disponibles en cada clase social o en cada grupo ocupacional que consideremos, las distintas tasas de natalidad en cada colectivo, los cambios en la jerarquía asignada a cada grupo ocupacional, cambios en el número de posiciones sociales hereditarias, etc.

Cada uno de estos procesos es importante en sí mismo. Así, el número de vacantes de un determinado estrato, nivel o grupo ocupacional no es constante a lo largo del tiempo, es más, lo normal es que varíe con cierta frecuencia; por ejemplo: el aumento de las actividades de servicios implica un aumento de las ocupaciones y de la clase de servicios de cuello blanco.

Otro efecto importante en las estructuras sociales modernas es la relativa disminución de las posiciones sociales hereditarias. A medida que aumentan las clases medias, los padres apenas tienen para legar a sus hijos poco más que una buena educación y la motivación para obtener una posición de estatus elevado. Una buena instrucción y educación formal es una de las mejores garantías del éxito ocupacional futuro. Conscientes de ello, las clases trabajadoras cada vez son más sensibles al proceso de educación formal de sus hijos. La mayor parte de estos factores son tenidos en cuenta en el modelo de desarrollo de Treiman (1970) ${ }^{1}$.

La motivación que tienen las personas para aspirar a posiciones sociales más elevadas de las que poseen en un momento determinado se convierte, por lo tanto, en un elemento importante para comprender la movilidad social. Esto se debe, fundamentalmente, a que la valoración que la sociedad tiene de una posición social concreta determina, en gran medida, la valoración que las personas se hacen de ellas mismas.

La movilidad social es, pues, el movimiento de individuos, familias y grupos de una posición social a otra. Es decir, pone en relación una posición social presente con otra pasada. Es un mecanismo para ver la proporción de personas que prospera en la estructura social y por qué motivos ocurre. Asimismo, es importante distinguir entre movilidad social vertical y horizontal. La movilidad horizontal es la que se produce cuando una persona cambia de lugar en la

1. Una versión en castellano del modelo de Treiman se encuentra en Gobernado (1994). 
estructura social sin cambiar de clase o de estatus social, por ejemplo: una persona de clase obrera cambia de empresa, o incluso un trabajador cambia de oficio sin que necesariamente cambie de estatus social. Otro tipo de movilidad es la vertical. Ésta se produce cuando el cambio supone variación de estatus, prestigio o clase social. A su vez, la movilidad social vertical puede ser entre generaciones diferentes, por ejemplo, entre la ocupación de los padres y la ocupación de los hijos o las hijas, entonces estamos hablando de movilidad social vertical intergeneracional. Por último, si la comparación la establecemos entre la primera ocupación de una persona y su ocupación actual, entonces se trata de movilidad social vertical intrageneracional. A efectos del estudio y del análisis de la estructura social, el tipo de movilidad más interesante es la movilidad vertical. A esta será a la que nosotros prestaremos atención aquí. Nos centraremos solo en la movilidad intergeneracional.

Antes de comenzar con el análisis de los procesos de movilidad propiamente dichos, es necesario hacer una breve mención expresa a la cuestión del uso o no de los varones como unidad de análisis. La gran mayoría de los estudios de movilidad social utilizan como unidades de análisis de la movilidad exclusivamente a varones. Esta circunstancia se debe fundamentalmente a que la distribución de las ocupaciones es diferente en los hombres que en las mujeres. Las ocupaciones en las mujeres se concentran fundamentalmente en las de cuello blanco de bajo nivel, al margen del rango ocupacional del que tengan sus padres. Por tanto, la estructura ocupacional de las mujeres está sesgada respecto al sexo (Hauser y Featherman, 1977).

Por otro lado, una comparación entre los varones y las mujeres muestra que las pautas de movilidad de los dos sexos son bastante similares. Es decir, cuando la comparación de las ocupaciones es con la de sus padres, las mujeres muestran una pauta de herencia y reclutamiento ocupacional similar al de los varones, aunque en el caso de las mujeres se da por vía de matrimonio (Kerbo, 1998). No obstante, los análisis de la movilidad en las mujeres presentan problemas relativos no solo a la falta de homogeneidad en la distribución de las ocupaciones, sino también a los mecanismos (por ejemplo: más importancia a la educación) y a las vías de movilidad (por ejemplo: se utiliza más la incorporación a la Administración pública). Es decir, tenemos que ser conscientes de los problemas que entraña el análisis de la movilidad de las mujeres ${ }^{2}$.

El desarrollo socioeconómico, por su parte, es un concepto relativo. Dicha relatividad hace referencia al hecho de comparar una posición o un territorio más desarrollado que otro. Por este motivo, el estudio del desarrollo cobra todo su sentido cuando se realiza un análisis comparado. Desde este punto de vista, aquí consideraremos dos territorios diferentes de un país desarrollado como

2. Para un análisis de los problemas que presenta la mujer como unidad de análisis en el análisis de clase en general, puede verse el trabajo muy bien documentado de R. Feito Alonso (1995), «Mujeres y análisis de clase», Revista Española de Investigaciones Sociológicas, 69, 149-171. 
es España, pero que se encuentran en diferentes niveles o estadios de desarrollo: Andalucía y Cataluña. En este artículo, trataremos de averiguar los cambios habidos en los procesos de movilidad social según la posición de estas diferentes comunidades autónomas en el proceso de desarrollo.

Está muy documentado cómo se pueden medir los procesos de desarrollo. Para ello, existen una gran cantidad de indicadores que ayudan a posicionar con claridad cual es el lugar que se ocupa en el proceso de desarrollo de una sociedad (Clark, 1967; Solé, 1976 y 1987; Bell, 1976; Wezel et al., 2003). Estos indicadores enmarcan cual es la posición relativa que ocupan tanto Andalucía como Cataluña en los dos momentos en el tiempo que consideramos: 1999 y 2004 . Asimismo, nos permite apreciar si se ha producido un proceso de convergencia o divergencia entre ambas comunidades autónomas a lo largo del lustro objeto de estudio.

Indicadores tales como el aumento de la esperanza de vida, el incremento del PIB, un mayor nivel educativo, una mayor tasa de población activa femenina, etcétera, son algunos de los que hemos tenido en cuenta. De estos indicadores, probablemente el más usado ha sido el de la disminución de la población activa dedicada al sector primario (Deutsch, 1961). Estos indicadores ponen de manifiesto qué sociedad y qué momento en el tiempo coinciden con situaciones estructurales de un mayor desarrollo social. Siguiendo estos indicadores para las dos comunidades autónomas con las que estamos trabajando, se puede observar su posición relativa en los dos momentos estudiados. A continuación, se puede observar cual es la posición respecto a estos indicadores en Andalucía y Cataluña en el lustro estudiado.

En la tabla 1, se muestra un conjunto diverso de indicadores de modernización. En todos ellos, se pone de manifiesto cómo las dos comunidades tienden a mejorar notablemente si se las compara entre el principio y el final del período considerado. Asimismo, cuando se compara a Andalucía con Cataluña, se observa que ésta última comunidad posee unos niveles bastante más altos que Andalucía en casi todos los indicadores, salvo en lo que se refiere a los años de estudios. En este caso, se comprueba el efecto de los sucesivos años de política educativa universalista que ha hecho que apenas existan diferencias de nivel educativo entre comunidades. Se ha producido un proceso de expansión educativa.

Según los datos de la tabla 1, los indicadores señalan que Andalucía se dirige hacia un mayor grado de modernización. Sin embargo, si consideramos el nivel de desarrollo por la velocidad con que Cataluña alcanza los niveles más altos, entonces se puede decir que esta comunidad lleva un ritmo más acelerado hacia el proceso de desarrollo.

El nivel de desarrollo en ambas comunidades, aunque es diferente, tiene cierto equilibrio. De los cinco indicadores propuestos, cada comunidad destaca en dos de ellos, si bien son distintos. Por un lado, Andalucía aumenta más en su PIB y disminuye en mayor proporción su porcentaje de población activa dedicada a la agricultura. Por otro lado, Cataluña aumenta en mayor proporción la población activa femenina y el nivel educativo. 
Tabla 1. Indicadores de desarrollo en Andalucía y Cataluña (1999-2004)

\begin{tabular}{|c|c|c|c|c|c|c|}
\hline & \multicolumn{3}{|l|}{ Andalucía } & \multicolumn{3}{|l|}{ Cataluña } \\
\hline & 1999 & 2004 & Diferencia & 1999 & 2004 & Diferencia \\
\hline $\begin{array}{l}\text { Esperanza de vida } \\
\text { al nacer }{ }^{1}\end{array}$ & 77,54 & 78,58 & 1,04 & 79,03 & 80,26 & 1,23 \\
\hline $\begin{array}{l}\text { \% Población } \\
\text { activa femenina }^{2}\end{array}$ & 36,58 & 40,48 & 3,90 & 44,68 & 51,00 & 6,32 \\
\hline $\begin{array}{l}\text { \% Población activa } \\
\text { agricultura }^{2}\end{array}$ & 13,33 & 11,44 & $-1,89$ & 3,11 & 2,27 & $-0,84$ \\
\hline PIB (millones de $€)^{3}$ & $68.653,51$ & $81.795,19$ & $13.141,68$ & $94.059,28$ & $106.527,60$ & $12.468,32$ \\
\hline $\begin{array}{l}\text { Media de años } \\
\text { de estudios }\end{array}$ & 9,03 & 9,47 & 0,44 & 9,06 & 10,08 & 1,02 \\
\hline
\end{tabular}

Fuentes:

1. INE. Indicadores demográficos básicos.

2. INE. Encuesta de población activa, segundos trimestres.

3. INE. Contabilidad regional de España.

4. MTAS. Encuesta de calidad de vida en el trabajo. Ocupados.

Elaboración propia.

\section{Movilidad intergeneracional y desarrollo: hipótesis}

La tradición de los estudios comparados en el análisis de la movilidad social es ya muy larga. Se remonta, al menos, a los trabajos de Inkeles y Rossi en 1956 o a los de Lipset y Zetterberg en 1963. Sin embargo, este método comparado es el mejor para estudiar los procesos de desarrollo: comparación en el tiempo y comparación entre sociedades.

Para nuestro análisis, seguiremos el modelo que Treiman (1970) elaboró para estudiar la tendencia de los procesos de herencia social y de influencia entre diferentes variables posicionales entre los padres y los hijos, según se trate de sociedades más o menos industrializadas y desarrolladas.

El análisis sociológico ha puesto de manifiesto que las sociedades industriales presentan tasas de movilidad relativamente altas. Los procesos de industrialización, urbanización y burocratización (Lipset y Bendix, 1963) fundamentan esta situación. El proceso de desarrollo facilita la tendencia a situaciones sociales de una mayor igualdad, con lo cual aumenta considerablemente la proporción de los estratos intermedios (Golthorpe, 1972). La estratificación social deja de tener una forma piramidal para adquirir una forma de rombo. Por otra parte, las sociedades que han alcanzado cierto nivel de desarrollo tienen tasas relativamente altas de movilidad social.

A continuación, proponemos un conjunto de hipótesis con las que estudiaremos cómo ha afectado el proceso de desarrollo y modernización a los procesos de movilidad en dos sociedades desarrolladas en dos momentos en el tiempo. 
La hipótesis primera $(\mathrm{H} 1)$ que proponemos para este trabajo se fundamenta en la tesis general de Lipset y Bendix (1963) y Golthorpe (1972). A partir de ella, se proponen otras cinco elaboradas a partir del modelo de Treiman (1970), relativas sobre todo a las diferentes modalidades de relación entre la posición social del padre y la posición social de los hijos.

H1. Si se acepta, como se ha visto en la tabla 1, que, según los indicadores de desarrollo considerados, Cataluña se encuentra en una posición más desarrollada y modernizada que Andalucía, y que el año 2004 es un momento en el tiempo de mayor desarrollo que 1999, entonces resultará que Cataluña, en 2004, tendrá una mayor tasa de movilidad que Andalucía.

Esta hipótesis se matiza con las que siguen, propuestas por Treiman (1970), y que aquí se comprueban para Andalucía y Cataluña:

H2. Cuanto más desarrollada es una sociedad, menor es la influencia directa del estatus ocupacional del padre en el estatus ocupacional del hijo.

H3. Cuanto más desarrollada es una sociedad, mayor es la influencia del nivel educativo en el estatus ocupacional.

H4. Cuanto más desarrollada es una sociedad, menor es la influencia del estatus ocupacional de los padres en la formación educativa de los hijos.

H5. Cuanto más desarrollada es una sociedad, más fuerte es la influencia de estatus ocupacional en la renta.

H6. Cuanto más desarrollada es una sociedad, menor es la influencia directa de la educación en la renta.

Estas hipótesis se han comprobado para muchas sociedades, sin embargo, el objetivo principal aquí es mostrar que no se cumplen de forma lineal para todas las sociedades. De hecho, funcionan de forma muy desigual según se trate de unas sociedades u otras. En el caso del análisis comparado entre Andalucía y Cataluña en los dos momentos estudiados, consideramos que estas hipótesis no se cumplen exactamente en todas las circunstancias.

\section{Datos, variables y método}

Estas hipótesis se probarán usando los datos de la primera (1999) y la sexta (2004) oleadas de la Encuesta de calidad de vida en el trabajo (ECVT). La ECVT es una encuesta que realiza el Ministerio de Trabajo y Asuntos Sociales, es de periodicidad anual y recoge información sustantiva sobre diversos aspectos relacionados con la situación laboral de los ocupados españoles. Proporciona información sobre las circunstancias que se producen en el lugar de trabajo; asimismo, hace referencia a la percepción personal que los trabajadores tienen del entorno y de las actividades que realizan en su profesión. Datos tales como: información sobre relaciones laborales; niveles de satisfacción sobre diferentes aspectos del trabajo; organización y división del trabajo; integración y promoción laboral; así como la situación ocupacional, familiar y los valores de los ocupados, entre otros. Se trata de un barómetro laboral sobre la 
situación de la calidad de vida en el trabajo que tienen los ocupados españoles. Además de todas estas cuestiones, la ECVT tiene un extenso módulo en el que se estudia la movilidad social en una generación ${ }^{3}$, que es el que aquí más nos interesa.

La ECVT está basada en un muestreo trietápico estratificado por comunidades autónomas y tamaño de municipio, cuyo ámbito ha sido toda España. El universo ha sido la población ocupada de dieciséis y más años de ambos sexos que reside en viviendas familiares. El tamaño muestral de cada oleada ha sido de $\mathrm{N}=6.020$ ocupados. Las entrevistas se han realizado en sus hogares para que los encuestados no estuvieran sometidos a posibles presiones derivadas del entorno de las empresas. El error de muestreo para el total nacional, en el caso más desfavorable $p=q=50$, y para un intervalo de confianza del 95,5 por 100 , ha sido de $\pm 1,4 \%$.

Las variables usadas en el estudio son aquellas que hacen referencia a las hipótesis estudiadas. Para la construcción de los procesos de movilidad social, se han usado las ocupaciones de los entrevistados y de sus padres. La ocupación presenta una gran ventaja en los estudios de movilidad, dado que intrínsecamente representan el nivel de estudios requerido para poder desempeñar una ocupación y, al mismo tiempo, suponen un determinado nivel de ingresos. En la ECVT, la ocupación se mide con tres dígitos según la clasificación nacional de ocupaciones de 1994 (CNO-94). El estatus de los individuos y de sus padres se ha medido de dos formas diferentes según cual sea el matiz que se necesite en la hipótesis de que se trate. Así, cuando se mide de forma objetiva, se calcula en base al índice de estatus socioeconómico (IES). El IES se ha construido de forma que varía de 0 a 100. Para ello, se ha seguido el esquema de Treiman (1970). Se ha calculado la media de ingresos que proporciona y la media de años de estudios que se necesita para desempeñar cada una de las ocupaciones de la CNO-94, convenientemente ponderadas (Requena, 2005). La fuente de error puede recaer en el hecho de haber utilizado la misma escala tanto para el entrevistado como para el padre del entrevistado. Dado que esta información no estaba recabada para los padres, se ha asumido que los ingresos de una misma ocupación serían, en términos medios, iguales para los entrevistados que para sus padres. De este modo, se puede usar este índice de estatus socioeconómico, tanto para los entrevistados como para sus padres, de forma que se pueda usar en los análisis de movilidad social ${ }^{4}$.

3. Los datos de la Encuesta de calidad de vida en el trabajo (ECVT) están disponibles, para los investigadores que la necesiten, en la Subdirección General de Estadísticas Sociales y Laborales del Ministerio de Trabajo (c/ María de Guzmán, 52. 28071 Madrid).

4. Este índice de estatus socioeconómico es sencillo de construir, al mismo tiempo que se puede usar con mucha facilidad en las investigaciones de estructura social comparada entre países o regiones, ya que permite un procedimiento estandarizado para escalar el estatus ocupacional. Es posible poner las ocupaciones en una escala según su localización en una jerarquía de recursos y recompensas. Este índice ya se ha usado con mucho éxito en investigaciones anteriores como las del propio Treiman (1970), Gobernado (1996) y Requena (2005). 
Cuando las necesidades de la medida del estatus se dan de una forma más reputacional, entonces se realiza el índice de prestigio Presca ${ }^{5}$, que está basado en una encuesta sobre el prestigio supuesto de las ocupaciones a tres dígitos de la CNO-94. Estas variables se han usado principalmente en el caso del estudio del estatus de los encuestados y sus padres.

El nivel educativo se ha medido en años de estudios finalizados por el entrevistado. Los datos que se exponen en los resultados hacen referencia a los valores medios para cada ocupación o para cada colectivo objeto de estudio, según sea el caso.

El nivel de renta se refiere a los ingresos mensuales netos calculados en euros. La variable referente a los ingresos se ha medido con las marcas de clase de cada intervalo de ingresos que medía la ECVT.

La tabla 2 muestra los valores máximos, mínimos, medios y las desviaciones típicas de estas variables para la media de los años 1999-2004, tanto para Andalucía como para Cataluña.

La estrategia analítica seguida en este trabajo ha consistido en elaborar correlaciones bivariadas entre las variables representativas, al objeto de confirmar o falsear las hipótesis propuestas para las dos comunidades autónomas estudiadas. Se trata de analizar el nivel de relación que existe entre dos variables que miden situaciones posicionales concretas, tanto para los entrevistados como para la generación inmediatamente anterior, es decir, la de sus padres. Las hipótesis propuestas hacen referencia a cómo incide una situación en otra; por ejemplo: el estatus ocupacional en la renta. En este caso, la estrategia ha sido ver el nivel de correlación entre estas dos variables y establecer si dicha comparación se ha modificado, tanto entre las dos comunidades como en los dos períodos estudiados, así como ver cual ha sido su tendencia en dicha variación.

Asimismo, se han establecido diferencias según el sexo de los entrevistados, de forma que se puede realizar no sólo la verificación de las hipótesis propuestas, sino que también es posible matizarlas en función de que se trate de varones o mujeres. Esta estrategia nos va a permitir observar con nitidez los procesos de variación en los cambios sociales relativos a los procesos de movilidad en relación con el desarrollo que ha tenido lugar en estas dos comunidades españolas en el último lustro, en función del género.

5. Esta escala se basa en una encuesta realizada en el año 1995 por el CIS. El universo de la muestra estaba formado por la población española de dieciocho y más años. El tamaño muestral era $\mathrm{N}=2.500$, con un muestreo polietápico estratificado por conglomerados, con selección de las unidades primarias de muestreo (municipios) de forma aleatoria proporcional, de las unidades secundarias (secciones) de forma aleatoria simple y de las unidades últimas de muestreo (individuos) por rutas aleatorias y cuotas de sexo y edad. El error muestral, para un nivel de confianza del 95,5 por 100 y $p=q=50$ fue de \pm 2 para el conjunto de la muestra. Véase Carabaña y Gómez Bueno (1996: 65). Los resultados de las puntuaciones derivadas de esta encuesta se han aplicado a las ocupaciones señaladas por los entrevistados de las ECVT según la CNO-94, con un nivel de desagregación de tres dígitos. 
Tabla 2. Descripción de las variables clave utilizadas en los análisis (años 1999-2004)

\begin{tabular}{|c|c|c|c|c|c|}
\hline Andalucía & $\mathrm{N}$ & Mínimo & Máximo & Media & Desv. típ. \\
\hline $\begin{array}{l}\text { Índice de estatus socioeconómico } \\
\text { del entrevistado }\end{array}$ & 1.832 & 18,89 & 64,21 & 32,43 & 9,79 \\
\hline $\begin{array}{l}\text { Îndice de estatus socioeconómico } \\
\text { del padre }\end{array}$ & 1.832 & 15,08 & 57,59 & 23,09 & 7,95 \\
\hline Prestigio Presca del entrevistado & 1.824 & 46,77 & 266,23 & 104,37 & 38,45 \\
\hline Años de estudio entrevistado & 1.813 & 0,00 & 22,00 & 9,26 & 4,42 \\
\hline Ingreso mensual neto & 1.481 & 135,23 & $4.507,59$ & 988,40 & 638,61 \\
\hline Cataluña & $\mathrm{N}$ & Mínimo & Máximo & Media & Desv. típ. \\
\hline $\begin{array}{l}\text { Índice de estatus socioeconómico } \\
\text { del entrevistado }\end{array}$ & 2.086 & 20,59 & 78,96 & 33,42 & 9,33 \\
\hline $\begin{array}{l}\text { Indice de estatus socioeconómico } \\
\text { del padre }\end{array}$ & 2.087 & 15,08 & 57,59 & 24,04 & 7,58 \\
\hline Prestigio Presca del entrevistado & 2.070 & 23,58 & 269,15 & 107,32 & 35,95 \\
\hline Años de estudio entrevistado & 2.044 & 0,00 & 22,00 & 9,57 & 4,26 \\
\hline Ingreso mensual neto & 1.792 & 135,23 & $4.507,59$ & $1.030,32$ & 545,03 \\
\hline
\end{tabular}

Fuente: ECVT de los años 1999 y 2004. Elaboración propia.

\section{Hallazgos empíricos}

El interrogante propuesto por la primera hipótesis (H1) se comprueba en la tabla 3. A continuación, se puede observar la tendencia hacia una mayor o menor tasa de movilidad según los diversos territorios, clasificados según el nivel de desarrollo de cada uno.

De acuerdo con la H1, la tasa de movilidad intergeneracional será mayor en Cataluña que en Andalucía, puesto que, si nos fijamos en la tabla 1, vemos que hay más desarrollo en Cataluña.

En la tabla 3, se puede comprobar que las tasas de movilidad vertical aumentan conforme sube el nivel de desarrollo, entendiendo que la fecha más reciente significa un mayor desarrollo que la fecha anterior. En los varones de Andalucía, dicha movilidad aumenta 4,7 puntos porcentuales de 1999 a 2004, mientras que en las mujeres la subida en el índice de movilidad es de 6,3 puntos porcentuales.

En el caso de Cataluña, el aumento de la movilidad de los varones es 8,1 puntos entre 1999 y 2004, y en el caso de las mujeres, la variación es solo de 0,8 puntos porcentuales entre las dos fechas que estamos considerando.

Por otro lado, si consideramos el argumento de Sen y Grown (1995), que proponen que cuanta mayor sea la diferencia entre los sexos menor es el desarrollo del país, vemos que los procesos de movilidad diferencial por sexos difieren bastante, tanto en el caso de Andalucía como en el de Cataluña. Incluso 
Tabla 3. Movilidad intergeneracional y desarrollo en Andalucía y Cataluña (19992004). Porcentajes respecto del total de la población ocupada

\begin{tabular}{|c|c|c|c|c|}
\hline & \multicolumn{2}{|c|}{ Andalucía } & \multicolumn{2}{|c|}{ Cataluña } \\
\hline & 1999 & 2004 & 1999 & 2004 \\
\hline \multicolumn{5}{|l|}{ Varones } \\
\hline Movilidad social vertical & 68,1 & 72,8 & 65,3 & 73,4 \\
\hline Ascienden & 41,9 & 43,3 & 34,7 & 38,0 \\
\hline Descienden & 26,2 & 29,5 & 30,6 & 35,4 \\
\hline \multicolumn{5}{|l|}{ Mujeres } \\
\hline Movilidad social vertical & 72,0 & 78,3 & 78,6 & 79,4 \\
\hline Ascienden & 41,6 & 44,8 & 49,7 & 43,9 \\
\hline Descienden & 30,4 & 33,4 & 28,9 & 35,5 \\
\hline \multicolumn{5}{|c|}{ Diferencias entre varones y mujeres } \\
\hline Movilidad social vertical & 3,9 & 5,5 & 13,3 & 6 \\
\hline Ascienden & $-0,3$ & 1,5 & 15 & 5,9 \\
\hline Descienden & 4,2 & 3,9 & $-1,7$ & 0,1 \\
\hline
\end{tabular}

Fuente: ECVT 1999 y 2004. Elaboración propia.

en esta última comunidad las diferencias son mayores que en Andalucía. Esta situación pone de manifiesto que las afirmaciones generales respecto a los procesos de desarrollo son arriesgadas cuando pretenden suponer generalizaciones muy amplias, lo que nos lleva a pensar que los procesos de desarrollo se caracterizan por su particularidad. Sin embargo, observando el apartado de diferencias entre varones y mujeres de la tabla 3, se observa que las distancias entre los géneros en Andalucía van en aumento conforme pasa el tiempo (3,9 frente a 5,5), mientras que en Cataluña la tendencia es hacia una disminución (13,3 frente a 6). Dicha tendencia puede entenderse como un avance hacia el desarrollo mayor que en Andalucía.

Todas las demás hipótesis, desde la segunda hasta la sexta, hacen mención estrictamente a la relación entre estratificación y desarrollo. Para la medida de estas hipótesis, se ha realizado un análisis de las correlaciones entre las variables implicadas, entendiendo que una mayor fuerza de la correlación equivale a una mayor implicación de un elemento de la estratificación social sobre otro.

Hipótesis 2. Cuanto más desarrollada es una sociedad, menor es la influencia directa del estatus ocupacional del padre en el estatus ocupacional del hijo.

Esta situación se ha medido utilizando como variables el índice de estatus socioeconómico del padre y del hijo. Para el caso de Andalucía y Cataluña, los datos son los siguientes:

Como se puede comprobar en la tabla 4, la hipótesis de la pérdida de fuerza del estatus ocupacional del padre en el del hijo se ve con claridad en ambas 
Tabla 4. Correlación entre el índice de estatus socioeconómico del padre y del hijo (Andalucía y Cataluña 1999-2004)

\begin{tabular}{lllllll}
\hline & 1999 & & & 2004 & & \\
\cline { 2 - 3 } & Varones & Mujeres & & Varones & Mujeres & Cumplimiento de la hipótesis 2 \\
\hline Andalucía & $0,507^{* *}$ & $0,443^{* *}$ & & $0,315^{* *}$ & $0,350^{* *}$ & Se cumple \\
Cataluña & $0,362^{* *}$ & $0,273^{* *}$ & & $0,295^{* *}$ & $0,203^{* *}$ & Se cumple \\
\hline
\end{tabular}

** Significación $p<0,01$.

Fuente: ECVT 1999 y 2004. Elaboración propia.

comunidades estudiadas. En Andalucía, la tendencia de la fuerza de la correlación en los años 1999 y 2004 ha disminuido tanto en los varones como en las mujeres, aun siendo mucho mayor el descenso en los varones. Lo mismo ocurre en el caso de Cataluña, aunque las disminuciones son proporcionalmente menores que en Andalucía, pero, no obstante, también se cumple la hipótesis 2 propuesta. En lo que respecta a esta hipótesis, no existen, pues, diferencias sustantivas entre ambas comunidades autónomas. Sin embargo, los datos confirman, de nuevo, la mayor situación de desarrollo de Cataluña frente a Andalucía, en lo que respecta a la fuerza de relación entre el estatus ocupacional del padre y el de los hijos.

Las hipótesis tercera, cuarta y sexta tienen que ver con el proceso de expansión educativa. Este proceso consiste en que cada vez hay más población que estudia y que, además, estudia durante más años. En la medida en que asciende la media de escolaridad entre la población, el nivel educativo se independiza del origen social, de la ocupación de llegada y, por supuesto, de la renta que se obtiene. Esto es así porque la educación aumenta, mientras que la estructura ocupacional y la distribución de la renta apenas varían (Boudon, 1977; Thurow, 1977). Treiman no tuvo en cuenta tal proceso.

Hay otro proceso no tenido en cuenta por Treiman y que dificulta el análisis que sigue: el aumento de las desigualdades internas de los países desarrollados. Los ingresos de las categorías ocupacionales más altas crecen mucho más deprisa que el resto, que las más bajas. Sin embargo, los ingresos de las inferiores no varían (Goldthorpe, 2007). Las desigualdades ocupacionales vuelven a tener una gran importancia en la estructuración de las sociedades avanzadas.

Hipótesis 3. Cuanto más desarrollada es una sociedad, mayor es la influencia del nivel educativo en el estatus ocupacional.

Para medir esta influencia, se ha utilizado el estatus de la ocupación medido por el prestigio Presca ${ }^{6}$ correspondiente a cada ocupación. Por otro lado,

6. El prestigio de la ocupación se trata del prestigio reputacional medido por la escala Presca realizada por el CIS en 1995, véase Carabaña y Gómez Bueno (1996). 
Tabla 5. Correlación entre el estatus ocupacional y el nivel educativo (Andalucía y Cataluña 1999-2004)

\begin{tabular}{llllllll}
\hline & 1999 & & & 2004 & & \\
\cline { 2 - 3 } & Varones & Mujeres & & Varones & Mujeres & & Cumplimiento de la hipótesis 3 \\
\hline Andalucía & $0,626^{* *}$ & $0,657^{* *}$ & & $0,502^{* *}$ & $0,439^{* *}$ & No se cumple \\
Cataluña & $0,487^{* *}$ & $0,512^{* *}$ & & $0,546^{* *}$ & $0,430^{* *}$ & Solo se cumple para los varones \\
\hline
\end{tabular}

** Significación $p<0,01$.

Fuente: ECVT 1999 y 2004. Elaboración propia.

para la medición del nivel educativo, se ha utilizado el número de años de estudios realizados por el individuo.

Como se puede observar en la tabla 5, la hipótesis 3, en general no se cumple. Solo en el caso de los varones de Cataluña se puede apreciar un aumento en la fuerza de la correlación ente educación y ocupación, que pasa de $r=0,487$ a $r=0,546$. En el resto de los casos, la influencia entre educación y prestigio ocupacional disminuye con el desarrollo en las comunidades estudiadas. A juzgar por los datos, se puede decir que sólo los varones de Cataluña siguen la pauta de la hipótesis propuesta por Treiman de tender hacia el aumento de la influencia del nivel educativo sobre el estatus ocupacional. En el caso de Andalucía, no se cumple en absoluto. La causa no es tanto un menor nivel de desarrollo, cuanto un acusado proceso de expansión educativa en Andalucía.

La propuesta de Treiman (H3) solo se cumple, en realidad, para los varones de Cataluña, lo que puede significar que solo en este colectivo es donde verdaderamente se da la tendencia indicada de una mayor influencia de nivel educativo en el estatus ocupacional. Es decir, este caso sería el verdaderamente desarrollado desde el punto de vista de Treiman. En el caso de las mujeres catalanas y en Andalucía es donde se ha producido el proceso de expansión educativa mayor, aunque ello no signifique que dicha expansión afecte a la distribución de las ocupaciones. De hecho, según la Encuesta anual de estructura salarial del INE, entre los años 1995 y 2004, se pone de manifiesto que las diferencias de la desigualdad de los salarios entre las categorías ocupacionales aumentaron, en dicho período, en un $60 \%$ en Cataluña frente a un $15 \%$ en Andalucía.

Hipótesis 4. Cuanto más desarrollada es una sociedad, menor es la influencia del estatus ocupacional de los padres en la formación educativa de los hijos.

Para medir esta relación, se ha realizado una correlación entre el índice de estatus socioeconómico de los padres y el número de años de estudio de los hijos.

Tal como se aprecia en la tabla 6 , la hipótesis 4 solo se cumple parcialmente. En el caso de Andalucía, la tendencia temporal es que se produce una disminución de la fuerza de la relación entre el estatus del padre y la educación de los hijos, sean éstos varones o mujeres. En cambio, para Cataluña, la ten- 
Tabla 6. Correlación entre el índice de estatus socioeconómico del padre y el nivel educativo del hijo (Andalucía y Cataluña 1999-2004)

\begin{tabular}{lllllll}
\hline & 1999 & & & 2004 & & \\
\cline { 2 - 3 } & Varones & Mujeres & & Varones & Mujeres & Cumplimiento de la hipótesis 4 \\
\hline Andalucía & $0,463^{* *}$ & $0,466^{* *}$ & & $0,420^{* *}$ & $0,447^{* *}$ & Se cumple \\
Cataluña & $0,421^{* *}$ & $0,300^{* *}$ & & $0,343^{* *}$ & $0,362^{* *}$ & Solo se cumple para los varones \\
\hline
\end{tabular}

** Significación $p<0,01$.

Fuente: ECVT 1999 y 2004. Elaboración propia.

dencia hacia una menor fuerza de tal relación es solo válida para los varones y no para las mujeres. En el caso de las mujeres, todavía había cierta relación de fuerza entre el estatus del padre y el nivel educativo de la hija. Sin embargo, en valores absolutos, esta fuerza es menor que en Andalucía.

Hipótesis 5. Cuanto más desarrollada es una sociedad, más fuerte es la influencia del estatus ocupacional en la renta.

Esta influencia se ha medido con la correlación entre el nivel de prestigio de la ocupación y la renta que alcanza el individuo.

Del mismo modo que en los casos anteriores, tal como se aprecia en la tabla 7, la hipótesis 5 se cumple en parte. La trayectoria temporal solo implica un aumento de la fuerza del estatus ocupacional en la renta en el caso de los varones y no en las mujeres, ya sea en una u otra comunidad autónoma. Sin embargo, independientemente de ello, la fuerza de dicha relación comienza siendo más alta en Andalucía, aunque termina siéndolo en Cataluña. En el caso de las mujeres, se aprecia un claro descenso en la relación propuesta, es decir, conforme pasa el tiempo, tanto en Andalucía como en Cataluña se produce una menor influencia de la ocupación en la renta.

Hipótesis 6. Cuanto más desarrollada es una sociedad, menor es la influencia directa de la educación en la renta.

Esta influencia se ha medido con la correlación entre la variable años de estudio del encuestado y la variable nivel de renta medido por los ingresos obtenidos.

Tabla 7. Correlación entre el estatus ocupacional y el nivel de renta (Andalucía y Cataluña 1999-2004)

\begin{tabular}{|c|c|c|c|c|c|}
\hline & 1999 & & 2004 & & \\
\hline & Varones & Mujeres & Varones & Mujeres & Cumplimiento de la hipótesis 5 \\
\hline Andalucía & $0,350^{* *}$ & $0,552^{* *}$ & $0,364^{* *}$ & $0,496^{* *}$ & Solo se cumple para los varones \\
\hline Cataluña & $0,307^{* *}$ & $0,363^{* *}$ & $0,436^{* *}$ & $0,343^{* *}$ & Solo se cumple para los varones \\
\hline
\end{tabular}

** Significación $p<0,01$.

Fuente: ECVT 1999 y 2004. Elaboración propia. 
La hipótesis 6 vuelve a cumplirse solo parcialmente. En la tabla 8, se observa que, en el caso de Andalucía, no se cumple en absoluto. Es decir, la trayectoria temporal no solo no implica una reducción, sino que se produce un aumento de la fuerza de la relación entre la educación y la renta, tanto en los varones como en las mujeres. Por otro lado, en el caso de Cataluña, dicha

Tabla 8. Correlación entre los años de estudio y el nivel de renta (Andalucía y Cataluña 1999-2004)

\begin{tabular}{llllllll}
\hline & 1999 & & & 2004 & & \\
\cline { 2 - 3 } & Varones & Mujeres & & Varones & Mujeres & & Cumplimiento de la hipótesis 6 \\
\hline Andalucía & $0,347^{* *}$ & $0,480^{* *}$ & & $0,509^{* *}$ & $0,530^{* *}$ & & No se cumple \\
Cataluña & $0,218^{* *}$ & $0,263^{* *}$ & & $0,339^{* *}$ & $0,247^{* *}$ & & Solo se cumple para las mujeres \\
\hline
\end{tabular}

** Significación $p<0,01$.

Fuente: ECVT 1999 y 2004. Elaboración propia.

Tabla 9. Distribución de los sectores de producción de la nueva economía* y herencia intergeneracional del sector de producción (Andalucía y Cataluña 1999-2004, en porcentajes)

\begin{tabular}{lrrrrrr}
\hline & \multicolumn{3}{c}{ Andalucía } & & \multicolumn{3}{c}{ Cataluña } \\
\cline { 2 - 3 } Población ocupada en.... & 1999 & 2004 & & 1999 & \multicolumn{2}{c}{2004} \\
\hline Agricultura y pesca & 12,23 & 9,48 & & 2,55 & 4,56 \\
Industria & 23,75 & 26,12 & & 35,88 & 36,48 \\
Servicios tradicionales & 41,92 & 40,08 & & 44,14 & 31,81 \\
Servicios de la nueva economía & 6,77 & 10,76 & & 8,77 & 13,37 \\
Servicios de la sociedad del conocimiento & 15,32 & 13,57 & & 8,66 & 13,78 \\
\cline { 1 - 2 } & 100,00 & 100,00 & & 100,00 & 100,00 \\
\hline Heredan sector & 38,84 & 35,08 & & 40,37 & 38,96 \\
\hline
\end{tabular}

Fuente: ECVT 1999 y 2004. Elaboración propia.

* Los subsectores de los servicios se han realizado incluyendo las siguientes ramas de actividad a dos dígitos de la clasificación nacional de actividades económicas de 1993 (CNAE-93):

- Servicios tradicionales: $(50+51+52+55+60+61+70+71+75+90+93+95+99)$ venta y reparación de vehículos; venta de combustible; comercio al por mayor, intermedio y comercio al por menor; reparaciones domésticas; hostelería; transporte terrestre y por tubería; transporte marítimo y fluvial; inmobiliarias; alquiler de bienes muebles; Administración pública; defensa seguridad social; organismos extraterritoriales; actividades diversas de servicios personales; hogares que emplean a personal doméstico.

- Servicios de la nueva economía: $(62+63+64+65+66+67+74+91)$ transporte aéreo y espacial; actividades anexas a transportes y comunicaciones; instituciones financieras y seguros; otras actividades empresariales; actividades asociativas.

- Servicios de la sociedad de la información y del conocimiento: $(72+73+80+85+92)$ actividades informáticas, investigación y desarrollo; educación; actividades sanitarias, veterinarias y servicios sociales; actividades recreativas, culturales y deportivas. 
reducción de implicación solo se produce en las mujeres. Aunque hay que señalar que, aunque en esta comunidad, la trayectoria temporal en los varones es hacia una mayor implicación de la educación en la renta, los valores absolutos de dicha implicación son mucho menores que en Andalucía.

Como complemento a la hipótesis primera, que hace referencia a la situación general del desarrollo y los procesos de movilidad. Aquí se puede apreciar el proceso inverso a la movilidad, es decir, el de la herencia ocupacional. Para apreciar esta cuestión, hemos puesto la atención en la herencia del sector de producción. En la tabla 9, se puede apreciar que, en general, se produce una disminución de los procesos de herencia, ya se trate de los ocupados de Andalucía o de Cataluña. Esta disminución es de 3,76 puntos porcentuales en Andalucía, mientras que en Cataluña es de solo 1,41 puntos. Dicha situación parece indicar, en el caso de Cataluña, un cierto grado de estabilización en la movilidad social.

Como se ha visto, la ocupación actual del sujeto tiene importantes dependencias del origen familiar: educación y ocupación del padre inciden de forma

Tabla 10. Educación, estatus e ingresos medios (Andalucía y Cataluña 1999 y 2004)

\begin{tabular}{|c|c|c|c|c|c|c|c|c|}
\hline & \multicolumn{4}{|l|}{ Andalucía } & \multicolumn{4}{|l|}{ Cataluña } \\
\hline & \multicolumn{2}{|l|}{1999} & \multicolumn{2}{|l|}{2004} & \multicolumn{2}{|l|}{1999} & \multicolumn{2}{|l|}{2004} \\
\hline & Medias & Des. típ. & Medias & Des. típ. & Medias & Des. típ. & Medias & Des. típ. \\
\hline \multicolumn{9}{|l|}{ Varones } \\
\hline Años de estudio entrevistado & 8,4 & 4,7 & 9,0 & 3,9 & 8,9 & 4,2 & 9,7 & 4,1 \\
\hline $\begin{array}{l}\text { Îndice de estatus socioeconómico } \\
\text { del entrevistado }\end{array}$ & 32,0 & 9,7 & 31,9 & 9,3 & 32,8 & 8,3 & 33,1 & 9,8 \\
\hline Prestigio Presca del entrevistado & 104,6 & 39,5 & 105,0 & 40,4 & 107,9 & 35,9 & 105,7 & 38,1 \\
\hline Ingreso mensual neto & $167.438,8$ & $118.313,7$ & $190.230,3$ & $95.366,4$ & $176.952,7$ & $88.439,0$ & $209.419,8$ & $95.006,6$ \\
\hline \multicolumn{9}{|c|}{ (1) } \\
\hline Años de estudio entrevistado & 10,4 & 4,7 & 10,3 & 4,2 & 9,3 & 4,1 & 10,7 & 4,4 \\
\hline $\begin{array}{l}\text { Îndice de estatus socioeconómico } \\
\text { del entrevistado }\end{array}$ & 33,6 & 10,8 & 33,3 & 9,7 & 33,4 & 9,3 & 34,9 & 10,0 \\
\hline Prestigio Presca del entrevistado & 103,1 & 35,1 & 103,9 & 35,6 & 106,1 & 32,0 & 109,8 & 36,4 \\
\hline Ingreso mensual neto & $127.029,2$ & $98.314,4$ & $153.046,7$ & $92.366,2$ & $124.052,5$ & $69.812,8$ & $156.418,9$ & $81.723,3$ \\
\hline \multicolumn{9}{|c|}{ Diferencias de las medias entre varones y mujeres } \\
\hline Años de estudio entrevistado & $-2,0$ & & $-1,3$ & & $-0,4$ & & $-1,0$ & \\
\hline $\begin{array}{l}\text { Indice de estatus socioeconómico } \\
\text { del entrevistado }\end{array}$ & $-1,6$ & & $-1,4$ & & $-0,6$ & & $-1,8$ & \\
\hline Prestigio Presca del entrevistado & 1,5 & & 1,1 & & 1,8 & & $-4,1$ & \\
\hline Ingreso mensual neto & $40.409,6$ & & $37.183,6$ & & $52.900,2$ & & $53.000,9$ & \\
\hline
\end{tabular}

Fuente: ECVT 1999 y 2004. Elaboración propia. 
poderosa en la ocupación y en los ingresos futuros del individuo. Asimismo, como se está viendo en este artículo, estos procesos de dependencia del origen familiar varían en función del momento y del lugar de desarrollo en el que se encuentra una sociedad. De este modo, si se considera que Andalucía y Cataluña se encuentran en momentos de desarrollo diferentes, entonces se puede afirmar que el hecho de haber nacido en una comunidad u otra de España supone una mayor o menor ventaja adicional en lo que respecta a las oportunidades ocupacionales futuras, ya que éstas varían ampliamente, incluso tratándose del mismo país.

Las carreras ocupacionales no son las mismas según se produzcan en una zona u otra del país. De este modo, el simple hecho de haber nacido en Andalucía o en Cataluña implica tener mayor o menor educación, estatus e ingresos futuros. Así, en la tabla 10, se puede observar cuales son estas variables tanto para Andalucía como para Cataluña en ambos años. En ella se puede apreciar que, en efecto, en Cataluña, en el año 2004, es donde se producen los niveles más altos en todas las variables consideradas. Ya se trate de varones o de mujeres, las medias de estudios, estatus e ingresos son siempre mayores en esta comunidad autónoma. El hecho de nacer en Cataluña implica una mayor probabilidad de contar en el futuro con un estatus ocupacional mayor.

\section{Conclusiones}

A partir de los resultados precedentes, se pueden establecer como relevantes las siguientes conclusiones:

En primer lugar, todos los análisis realizados han resultado muy significativos desde el punto de vista estadístico. Todas las correlaciones bivariadas han mostrado una significación menor de 0,01 . Estos análisis han demostrado que los procesos de movilidad social varían de forma desigual en las dos comunidades y en los dos momentos estudiados.

En segundo lugar, se observa que los procesos de movilidad social, en su relación con los procesos de desarrollo, actúan de forma desigual según se trate de unas sociedades u otras. De los datos anteriores, se infiere que los procesos de movilidad social no tienen una trayectoria lineal hacia el desarrollo en todas las sociedades. De hecho, su funcionamiento es peculiar en cada una de ellas, aun pensando que se trata de sociedades que caminan por la misma línea, o similar, de desarrollo, dado que son comunidades de un mismo país.

De la misma forma que Inglehart (1998) demuestra que los procesos de cambio social no son lineales y que éstos varían en función de la sociedad de la que se trate, igual se puede argumentar con los procesos de desarrollo entre sociedades. Como se ha visto en los análisis anteriores, esta circunstancia se produce con el proceso de movilidad social. De hecho, la trayectoria propuesta por los clásicos como Lipset y Zetterberg (1963) habría que matizarla en fun- 
ción de cada sociedad concreta. El mismo argumento se puede utilizar en el caso de los grupos pertenecientes a una misma sociedad. Como se ha apreciado a lo largo de todo el texto, las variaciones entre los géneros son sustanciosas, por lo que no es lo mismo el funcionamiento, desde el punto de vista del desarrollo, de los procesos de movilidad social según se trate de varones o de mujeres. Esta situación se ha visto con claridad en el desarrollo de las diferentes hipótesis estudiadas en este artículo, y cómo una gran parte de ellas no se cumple de la forma exactamente predicha para alguna de las comunidades estudiadas.

Por último, no se puede decir que la movilidad social tienda a la convergencia o a la divergencia en las dos comunidades estudiadas. Como se ha visto en los análisis precedentes, no hay una línea o una trayectoria fija hacia la que apunten, simultáneamente, las dos comunidades. Esto nos lleva a pensar que la realidad social de ambas es lo suficientemente diferente como para que no sigan procesos similares en lo que respecta a la movilidad social; situación que se demuestra con las diferencias de verificación y falsación de las hipótesis enunciadas según se trate de Andalucía o Cataluña, o de varones o mujeres en estas comunidades.

\section{Referencias bibliográficas}

BELL, D. (1976). El advenimiento de la sociedad postindustrial. Madrid: Alianza.

BOUdON, R. (1977). «Education and social mobility: A structural model». En: KARABEL, J. y Halsey, H. (eds.). Power and ideology in education. Nueva York: Oxford University Press, 186-196.

CACHÓN, L. (1989). ¿Movilidad social o trayectorias de clase? Madrid: CIS.

Carabaña, J. (1997). «La movilidad social en España». En: VV.AA. Movilidad social e igualdad. Madrid: Argentaria / Visor.

- (1999). Dos estudios sobre movilidad intergeneracional. Madrid: Argentaria / Visor.

Carabaña, J. y Gómez Bueno, C. (1996). Escalas de prestigio profesional. Madrid: CIS.

ClarK, C. (1967). Las condiciones del progreso económico. Madrid: Alianza.

Deutsch, K. (1961). «Social movilization and political development». American Political Science Review, 55, 17-24.

ERIKSON, R. y GOLDTHORPE, J. (1992). The constant flux: A study of class mobility in industrial societies. Oxford: Clarendon.

Feito Alonso, R. (1995). «Mujeres y análisis de clase». Revista Española de Investigaciones Sociológicas, 69, 149-171.

GobernADO, R. (1994). "Modernidad y estratificación social: análisis comparativo de las estructuras sociales de Cataluña y Andalucía». Revista Española de Investigaciones Sociológicas, 67, 87-108.

- (1996). «El logro ocupacional en España veinte años después». REIS, 74, 209224.

Golthorpe, J.H. (1972). En: Bendix, R. y LIPSET, S.M. Clase, status y poder. Madrid: Euroamérica, III, 385-412. 
Golthorpe, J.H. (2007). "Globalisation and social class». En: On sociology. Stanford, Calif.: Stanford University Press, I, 91-116.

Hauser, R. y Featherman, D. (1977). The process of stratification. Nueva York: Academic Press.

INE (1993). Clasificación nacional de actividades económicas 1993. Madrid: INE.

- (1994). Clasificación nacional de ocupaciones 1994. Madrid: INE.

- (2004). Encuesta anual de estructura salarial. Madrid: INE.

INGLEHART, R. (1998). Modernización y postmodernización: El cambio cultural, económico y politico en 43 sociedades. Madrid: CIS.

InKELES, A. y Rossi, P.H. (1956). "Nacional comparisons of occupations prestige». American Journal of Sociology, 61 (1), 329-339.

Kerbo, H.R. (1998). Estratificación social y desigualdad. Madrid: McGraw Hill.

LIPSET, S.M. y BENDIX, R. (1963). Movilidad social en la sociedad industrial. Buenos Aires: Eudeba.

LIPSET, S.M. y ZETTERBERG, H.L. (1963). «La movilidad social en las sociedades industriales». En: LIPSET, S.M. y BENDIX, R. Movilidad social en la sociedad industrial. Buenos Aires: Eudeba, 27-92.

REQUENA, F. (2005). La estructura ocupacional española. Madrid: MTAS.

SEN, G. y GROWN, C. (1995). «Gender and class in development experience». En: Roberts, B.R.; Cushing, R.G. y Wood, C. (eds.) (1995). The sociology of development. Aldershot: Edgar Plublishing Co., 1, 395-430.

Solé, C. (1976). Modernización: un análisis sociológico. Barcelona: Península.

- (1987). Ensayos de teoría sociológica: Modernización y postmodernización. Madrid: Paraninfo.

ThUROW, L.C. (1977). «Education and economic equality». En: KARABEL, J. y HaLSEY, H. (eds.). Power and ideology in education. Nueva York: Oxford University Press, 325-334.

Treiman, D.J. (1970). «Industrialization and social stratification». En: LAUMANN, E.O. (ed.). Social stratification: Research and theory for the 1970's. Nueva York: The Bobbs-Merril Company, Inc., 207-234.

Wezel, Ch.; Inglehart, R. y Klingemann, H.D. (2003). "The theory of human development: A cross-cultural analysis». European Journal of Political Research, 42, 341-379. 